\title{
Cardiac Rhabdomyoma
}

National Cancer Institute

\section{Source}

National Cancer Institute. Cardiac Rhabdomyoma. NCI Thesaurus. Code C6739.

A well circumscribed benign tumor arising from cardiac muscle. It usually affects children and may be present in the fetus. Depending on tumor location and size, cardiac, respiratory, and hemodynamic parameters may be affected. There is an association between cardiac rhabdomyoma and tuberous sclerosis. 